\title{
Exploring the Potential of Depictions with Sun Reflections
}

\author{
Patrick Tobias Fischer ${ }^{1}$, Eva Hornecker ${ }^{1}$, Johann Gielen*, Johannes Hartmann ${ }^{1}$, Marco \\ Schmandt ${ }^{1}$, Anna Rack ${ }^{1}$, Marie Bornemann ${ }^{1}$, Felix Dondera ${ }^{1}$, Herbert Grinda ${ }^{1}$ \\ 1: Bauhaus-Universität Weimar \\ Bauhausstr. 11, 99423 Weimar, Germany \\ \{FirstName.LastName\}@uni-weimar.de \\ * : TU Ilmenau, Lichttechnik \\ Prof.-Schmidt-Str. 26, 98693 Ilmenau, Germany \\ johanngielen@gmail.com
}

\begin{abstract}
In this paper we explore alternate projection and imaging mechanisms that don't rely on luminous sources, such as screens or LEDs. We investigate how sunlight can be used along a continuum ranging from non-informational aesthetic lighting to simple controlled reflections of sunlight that provide enough information to depict legible content. Rethinking the pixel as a reflection exposed challenges in mechanics, material and ease of control. We found that slow, precise movements work best. The threshold between pixel and non-pixel provides great potential to add additional aesthetics to a depiction based on sunlight, by transitioning between atmospheric ambient lighting and information representation. Our exploration of depictions without traditional information-rich content provides an alternative to think about ambient, calm and public displays.
\end{abstract}

\section{Author Keywords}

Light; pixel; reflection; media architecture; public interfaces; ambient interfaces.

\section{INTRODUCTION}

Technology for public displays and media architecture (for examples see [25]) typically employs projections, illumination technology, or embedded screens [11]. Critical voices argue that with permanent screen-based media façades attached to it, the building itself tends to dissolve and loose its identity [4], merely being a carrier of signs, but not enhancing the architecture [10]. People may get bored of these soon and may even reject them [19, 27]. But in lighting design, the medium of light itself is used as a design element contributing to architectural space $[17,21]$ to convey basic information (e.g. highlight architectural elements [15]), affect aesthetics or create atmosphere. Much can be learned from this field, which considers all controllable elements and aspects that influence the visual appearance of a space

Permission to make digital or hard copies of all or part of this work for personal or classroom use is granted without fee provided that copies are not made or distributed for profit or commercial advantage and that copies bear this notice and the full citation on the first page. Copyrights for components of this work owned by others than the author(s) must be honored. Abstracting with credit is permitted. To copy otherwise, or republish, to post on servers or to redistribute to lists, requires prior specific permission and/or a fee. Request permissions from Permissions@acm.org.

PerDis '15, June 10 - 12, 2015, Saarbruecken, Germany

Copyright is held by the owner/author(s). Publication rights licensed to ACM.

ACM 978-1-4503-3608-6/15/06 ..\$15.00

DOI: http://dx.doi.org/10.1145/2757710.2757712
[16], such as daylight, artificial light and the reflective and transmissive properties of surfaces and materials.

Inspired by this perspective and by examples of media architecture that integrate complex interactions with natural light and exploit reflections of ambient light, we aim to explore alternate projection and imaging mechanisms that don't rely on screens or LEDs, but are based on reflections of existing light sources, in particular natural light. We believe that such displays can blend into urban settings in a more subtle way than conventional screen-based media, while nevertheless offering a potent medium that users of recreational areas in public spaces appreciate for its subtlety. In particular, low-resolution content that influences the ambience of a space can harmonize with physical media architecture. We believe that it is this deep physical integration and potential calmness that can make this intriguing beyond brightness, contrast and resolution of the medium.

In the project presented here, we explored how we could use natural light sources, in particular the sun, to create projections and depictions. We studied the expressive and aesthetic potential of such projections, the degree of precision in imagery achievable, options for controlling these projections, and how these could be utilized to convey information. Using light itself as a material for design, our exploration is at the intersection between displays and lighting design and has as core question: can we create aesthetic, expressive and controllable depictions based on natural light projection? To generate and control such depictions, we need new forms of pixels that control the luminance of a surface by reflected light. A first step within our exploration was to create individual controllable pixels, followed by building a pixel matrix or set of pixels. These pixels are based on contrast between light reflections and shade.

We discuss outcomes of an interdisciplinary student project, which begins to explore the design space of controlled reflected light to produce projections based on visual contrast in luminance. The project was conducted in collaboration with a professional lighting designer and involved a group of students from media informatics and media architecture. For the first half of this five-month project, the team experimented with alternative mechanisms for creating and controlling light reflections. Then, a subset of these mechanisms was refined using rapid prototyping techniques to enable a higher level of control, and to create and experiment with pixel matrices and their aesthetic visual effects. 


\section{BACKGROUND}

Lighting design links the fields of visual perception, optics, architecture, art and technology [16, 17, 21]. It designs all controllable elements and aspects impacting the visual appearance of a space, including specs and positions of light sources, surface properties and natural light in a space [6]. It aims to achieve functionality and aesthetics appropriate for a space and its use(rs). The four main design elements are intensity, color, distribution, and movement.

A subset of Media Architecture creates highly innovative visual displays without relying on artificial light sources or directly changing the luminous flux or spectrum of the light source. Innovative and beautiful examples of such 'physical' Media Architecture, such as HypoSurface by Mark Goulthorpe can be found in [26, 9]. In the past, academic discussions referred to these types of designs as Kinetic Screens [20], Mechanical Media Façades [10, 11], Mechanical Displays [8] or Mechanical Pixels [26].

A pixel is defined as the "smallest addressable element" or controllable element of a picture [5, 18]. While in an idealized description a pixel is merely a point, in technical practice it may be a small quadrant, frayed dot (CRT monitors), consist of 3 mini squares (LCD), or a circle (LED matrix). Pixels thus do not need to be uniform. Using reflected natural light as our medium to depict information, we need to work with contrast instead of traditional pixel points. Contrast is created from juxtaposing bright and dark surfaces, or light and shade. An example of a display that relies entirely on contrast is Shade Pixel [13], a 7x11 pixel screen with an elastic skin pulled back by magnets. The resulting indentations result in a controllable matrix of shades. A primary question for our project was: What kind of light pixels can we create from surface reflection on a projection surface, and how much information) these will be able to convey.

We here focus on displays that are not merely based on mechanical principles, but rely on the reflection and transmission of light. Luminance measures the perceived brightness of objects. Beyond luminance, the photometrical parameters of transmissive and reflective spectra affect the resulting appearance, as they determine the perceived colour of a surface. When designing natural light projections, one of the key issues is how to achieve digital control of the visual appearance (luminance and/or color) of a surface. Being able to influence either one or both means being able to create individual pixels that are visually distinguishable from each other. In terms of optics this means creating contrast. There are various methods, based on optical principles, for influencing transparency, color and luminance of an object.

The angle of incidence of light hitting a reflective surface controls its luminance. Thus, moving either the light source, the surface, its angle, or the observer affects the resulting visual effect. An object seen from the same point of view can look brighter or darker when the position of the light source (e.g. sun) changes because different amounts of light are reflected into the viewers' eye. For example, we are

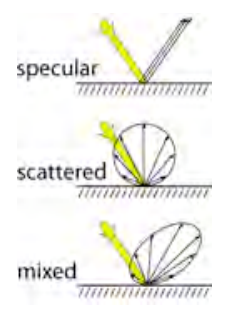

Figure 1. Types of reflection on a surface blinded by sun reflections in a window. Stepping away, we are not blinded anymore. Lighting design and architecture may use this effect to e.g. create a different impression of a room depending on which side it is entered from.

In media architecture, we need to be aware of these effects and to design for them, but usually cannot move the original light source or the viewer. But we can change the orientation of the surface, and thus affect the angle of reflection, thereby affecting light and shade contrasts for the viewer. Moreover, it is possible to change the color appearance of a surface as well as the color of transmitted and reflected light by tilting a surface, which manipulates the angle of incidence onto the material. Dichroic filters can block light or reflect only a specific spectrum, based on the principle of wave interference.

Another option is changing the reflective properties of a surface. The physics of light mean that a colored surface absorbs certain parts of the light spectrum and reflects others. Reflected light then contains different wavelengths than the original source - a colored reflection.

Moreover, the surface finish affects the reflection quality (figure 1), as when we clean and polish a window or wax wood. Basically, this is the distinction between a glossy and a matte surface. Incident light can be reflected specular (direct), scattered or as a mixture of both [10]. This depends on the surface finish of the material: matt, glossy or mixed. Thus, to change a reflection, one would need to change the surface quality. In fact, this phenomenon is more common than one might think. The appearance of a water surface can vary: without any waves, it has a glossy appearance, while when the surface is rippled it looks matt.

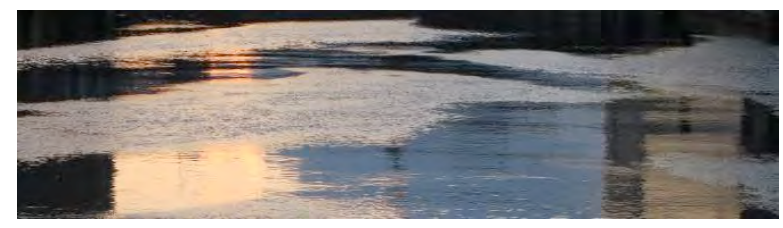

Figure 2. Different reflective properties of a water surface

Finally, it is technically possible to change the transmissive properties of a material, for example liquid crystal glass (LC-Glass) is transparent or translucent depending on whether an inner layer of liquid crystal is activated, and electrochromic glass changes the amount and color and light transmitted through it depending on a current applied.

\section{LIGHT REFLECTIONS AS EXPERIMENTAL MATERIAL}

Our experiments began with identifying means of creating single 'pixels' of light, exploring their aesthetic potential, and means of control. They proceeded via iteration of prototypes to improve control over pixel behavior and appearance and to control several pixels. The main aims were aes- 
thetic and the creation of a (small) vocabulary of light behaviors that can convey meaningful content. For light reflections, it turned out that a rather strong light source is needed for a clear visual effect. We thus usually used the sun, and, in its absence (weather) strong luminaires, such as overhead projectors.

We can roughly distinguish our explorations into two general approaches. One experiments with the angle of incidence, i.e. surface orientation, and utilizes mirrors to create distinct light pixels. A mirror reflects light specular (direct) from its surface. Its angle of incidence can be manipulated mechanically. The majority of our other explorations relates to controlling deformations of reflective materials in order to focus and diffuse incident light onto a projection surface. Our work focused on how to reliably create and control collections of such pixels, the aesthetic effects achieved, and its informational value.

\section{Surface Deformation Experiments}

In a series of experiments we explored mechanisms to affect the reflective properties of materials via deformation. Deformation affects surface finish, as it can scatter light and thus results in different types of reflections. Searching for non-rigid materials that withstand bending and deformation, we found space blankets, a thin PET film coated with a metallic reflecting agent (aluminum), usually in gold or silver, which reflects up to $97-99 \%$ of light. It has high tensile strength, dimensional stability and low gas permeability, can be stretched, folded and put under pressure. Spread flat, the material creates direct and diffuse reflections. Tiny crinkles result in effects similar to reflections on water. Space blankets seemed ideal for experiments, being cheap, and having suitable optical and mechanical properties.

PUMP MIRROR. An early exploration was the 'pump mirror', consisting of a space blanket wrapped tightly around a concave ring structure. When air is pumped out, the previ- ously flat blanket is sucked in, creating a concave mirror that focuses light into one point (figure 3 left). Pumping air in creates a convex mirror that diffuses light. Unfortunately, the mechanism turned out to be too complex and expensive for creating a pixel matrix. Each mirror would have needed a bidirectional pump and a pressure sensor as well as a loop control. Nevertheless, the prototype revealed at what point light reflections transition from random patterns to controlled and focused depictions. Mounting the foil around the structure resulted in tiny folds and variances in stretch, which creates rather unpredictable aura-like effects, as visible in figure 3 (left). When deflated, an aesthetic but random reflection is produced. Very high and very low pressures in the reflector create defined reflections, that, when arranged in a matrix, can convey information. This threshold between clear information and 'noise' marks the transition between aesthetical lighting and information depiction.

STAMP PIXEL(S). Learning from the prior prototype, we tried to shape the foil surface directly with pressure points at its back. Initially, felt pens were simply pushed and turned against the foil. However, reflections were too fuzzy and had no clear shape. Nonetheless, this exploration accidentally led to a promising approach. A role of sticky tape pressed against the foil creates a directed reflection with an evenly bright circle. If a finger is pressed against the foil inside this ring-stamp, the circle transitions into a bright ring that enlarges when more pressure is applied. This three-step mechanism was further developed into a 3D printed spring mechanism, actuated by a servo. To test how multiple of these mechanisms would perform behind one sheet of reflective PET foil, a module with three pixels was printed (figure 4). Due to the foil properties, the mechanism worked extremely well, creating repeatable shapes. Compared to an individual pixel, the module lost some aesthetical properties such as the random reflections of figure 3 (far right), but these can be reintroduced in future designs.
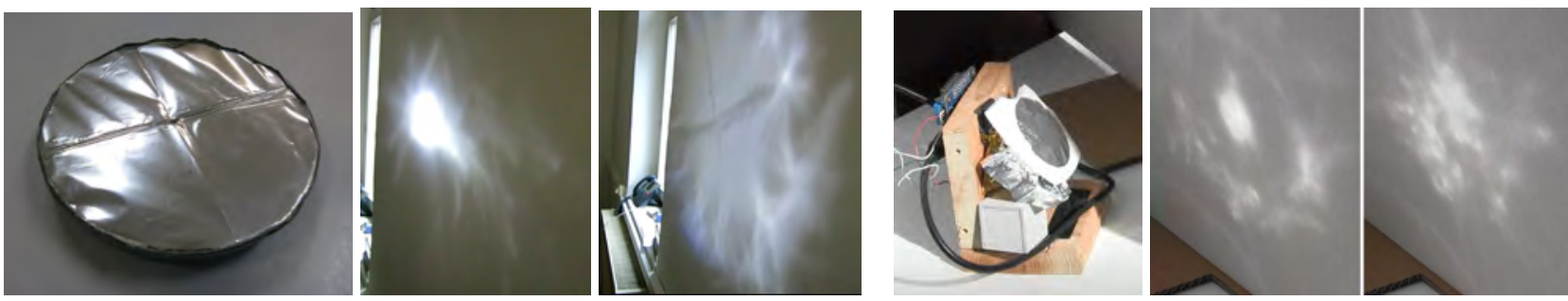

Figure 3. Pump mirror (left) and a single Stamp Pixel (right) each with their reflections (focused and random diffused)
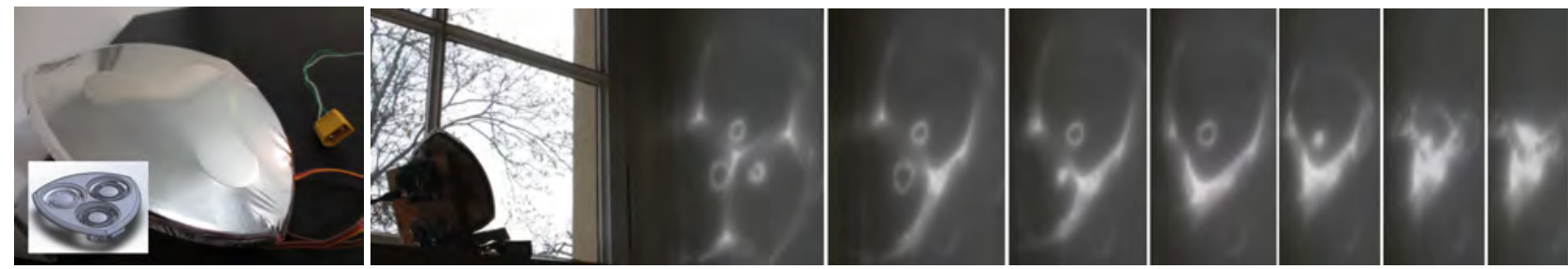

Figure 4. Refined Stamp Pixel 3-Matrix. From left: Mechanism (inlay: inner mechanism), stepping through the stages for each of the 3 pixels, where the inner ring reflection becomes a filled circle, then the outer ring contracts until it disappears into a dot. 


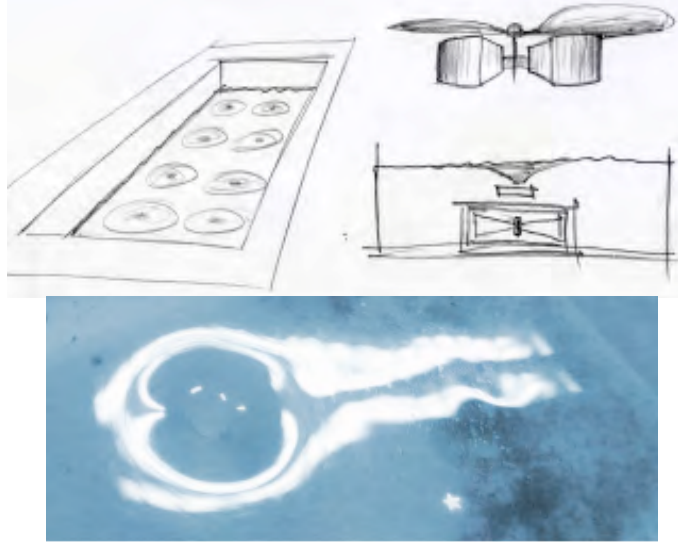

Figure 5. Sketch of a puddle vortex matrix and control motor. Bottom: photo of working prototype placed in a puddle.

Due to the simple mechanical mechanism, the module design is open for parametric generated shapes and has potential for arranging multiple pixels. Both pump mirror and stamp pixel produced aesthetic and intriguing reflections.

PUDDLE MATRIX. Another round of experiments went back to the original inspiration of reflections on a puddle surface. What if we could control these deliberately? The core idea was to create a vortex within the puddle that distorts reflections. On the bottom we would put a mat with integrated chambers that hold a magnetic stirrer creating an undertow or small maelstrom visible on the surface (figure 5). The biggest technical challenge is to create an actuator small enough to be invisible in shallow water. Typical puddles at the side of a street are $1.5-2.5 \mathrm{~cm}$ deep. Besides of waterproofing, floating particles, the mechanism size, and robustness posed challenges. A brushless motor with just enough torque was used in a low self-made casing $(<7 \mathrm{~mm})$. Technical feasibility was a challenge due to the physics of a vortex. The magnetic stirrer design, robust against particles due to magnetic bearing, was dropped because we could not balance a magnetic field so as to hold the stirrer in place. Instead, a plain bearing with a very loose axle was used to prevent the fan from jamming. Distorting the reflections in a puddle was an interesting idea, because of the background that the surroundings provide for the vortex effect, and how these effects could distort e.g. straight lines of the surroundings. Also, when walking by, one sees a reflection in the puddle for quite some time, but only at close, the ground becomes visible. Furthermore, a vortex is unexpected in a puddle and thus is an intriguing phenomenon.

ORIGAMI. Another experiment used Origami shapes for atmospheric output controlled by linear actuators. A first challenge is the material, which needs to be foldable, sufficiently flexible while rigid, and reflective. While interpretable as a connected set of moveable mirrors, in practice the approach of folding reflective material varies surface shape and thus type of reflection. Figure 6 shows some of our explorations. The resulting reflections resemble a water surface. Folds are not visible as borders or lines, but bundle the light. Each bundle of light is the result of an internal surface, and all of these move in response to manipulation of the origami form, but in random patterns. We were not able to create a fully controlled reflection, which means the individual highlights cannot be used as 'controllable light pixels'. A surprising effect of the folding technique was that reflections can be layered, one moving 'over' other reflections. The origami technique results in an organic and ambient display of aesthetic. But it has little informational value because individual light bundles cannot be controlled independently. Moreover, folding required extensive manual labor and thus does not scale easily for larger displays. Furthermore, repeated movement tends to damage the material. Overall, the experiments with surface deformation indicated that this type of reflection display is more suited to ambient displays aimed at creating and affecting atmosphere.

\section{Reflection Angle of Incidence: Controlling Mirrors}

Using mirrors, we get a clear specular reflection that can be moved by tilting the mirror. In a series of experiments, we explored how to steer and control light pixels from mirror reflections. An early experiment involved a matrix of mirror 'pixels' glued to blocks that could be shifted back and forth (figure 7 left). While the play of light dots on the floor was pleasing, this requires a high degree of precision to build. Slight deviations in how a mirror was attached had a big impact on the resulting pattern of reflections. Also, moving the blocks back and forth had little visual effect.

PAN-TILT. Attention thus shifted to reliable control of the orientation of individual mirrors. This resulted in the pantilt mirror, where two servo motors each control one axis.
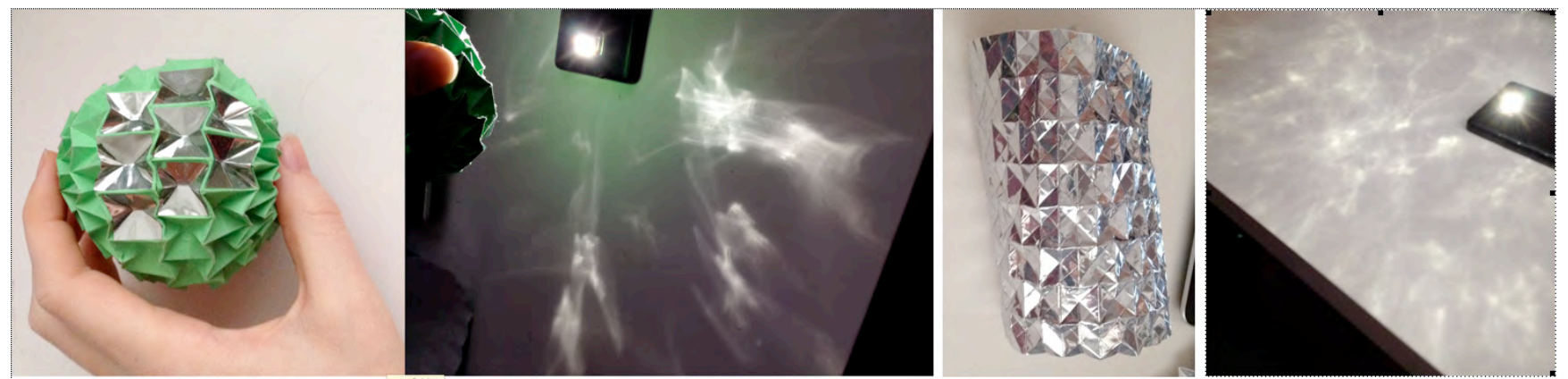

Figure 6. Top: Origami ball and reflections. Bottom: Folded pattern and resulting reflections 

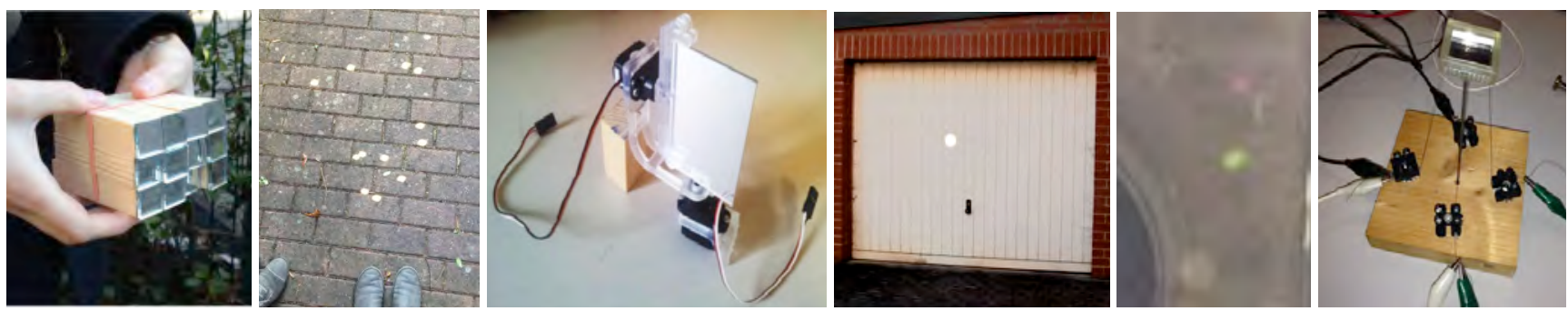

Figure 7. Left: Matrix of mirror pixels and its reflections on the ground. Middle: first Pan-Tilt mirror prototype and resulting projection onto a garage. Right: Reflections from several pan-tilts with colored mirrors. Far right: pan-tilt with Nitinol wire control

This is inspired by pan-tilt steering in the movie industry. It created a clear and reliably controlled projection. But servo motors can only move step by step, which results in jerky movements of the light points. The further away the projection surface, the stronger this effect, as distance potentiates the angle of movement. We thus looked for other means of control that could create smoother movement. Nitinol wire emerged as a potential solution. Heated up, it contracts, and thus can be used as actuator. We built a small experimental prototype (figure 7 right), and learned that while Nitinol reacts quickly to activation, it needs time to relax. Moreover, the wire only shortens by around $4 \%$, limiting the maximum possible change in orientation. Also, it was unclear how to reliably stop at defined angles. The precision needed to manufacture such a mechanism was too high, in particular, as the mechanism needs to be rigid and strong to exploit shortening of the wire in a reliable way.

Thus, while this approach was technologically interesting and created very smooth movements, it was not a viable solution. We therefore returned to the pan-tilt approach, adapting it with a transmission rod between servomotor and mirror, which generates smoother movements. With this, it should be possible to implement a pong-game on urban facades (cf. [3]), to follow people with the projected light dot, or other kinds of games in an urban environment. Once we experimented with several mirrors, it became difficult to follow and distinguish several moving light dots. We thus extended the approach with colored mirrors for different colors of reflections, which increases the display's legibility and visual interest, as colored reflections are less common in nature. An unexpected phenomenon appeared when we pursued a design with several mirrors, testing over several

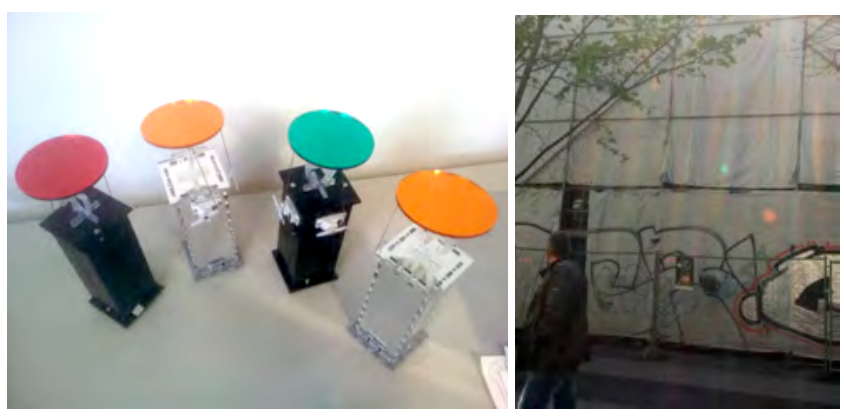

Figure 8. Our motorized mirrors. Man (left) looking up at light pixels (orange behind tree, green and red above graffiti). days which colors most reliably produce a bright reflection. This was not consistent; instead differently colored mirrors could produce bright reflections one day and be dull the next day. The reason is that depending on weather, sun position and location on earth, different spectra of sunlight arrive on the ground. The colored light reflected from a colored mirror has already been filtered in the hemisphere.

Further experimentation demonstrated that the pan-tilt approach can project at a wide panning angle (up to $360^{\circ}$ ). Figure 9 shows a test setting. Indicated in red are areas of building façades, which can be used for sun reflections controlled by a Pan-Tilt mechanism (red circle in top center).

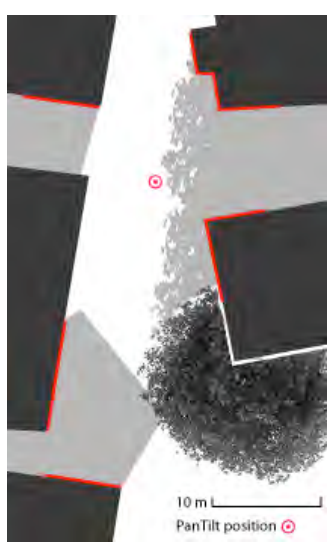

Figure 9. Facades usable for reflections from a $360^{\circ}$ Pan-Tilt mirror. Content can thus be distributed over a fairly wide area (by moving it). Reflections can only occur within shaded areas (light grey).

Similar to the Stamp Pixel Matrix, we created a more extended prototype based on the pan-tilt approach to explore how it could be used for a light installation. Advantages of the pan-tilt mechanism are that it can easily be scaled up to larger numbers of pixels by building more motorized mirrors, and provides a good level of control. For teaching purposes, the student project was to extend this into a larger installation prototype with distributed control, but we here only focus on the core mechanism relevant to this paper. Five coloured pan-tilt mirrors were built, of which two could be directly controlled. The other three react to the vicinity of another light pixel, controlled by our server, jumping around or drawing a sine wave. Two servo motors, controlled by a panSTamp, steer a mirror that creates a light pixel. With our final prototype, laser-cut acrylic boxes hide the electronics (figure 8, left). We tested this system in-situ in a pedestrian zone, using a building covered with tarpaulin for building works as projection plane (see figure 8). While [3]'s pink solar installation employs image recognition, our system relied on calibration to ensure pixels remain on the facade and to calculate pixel vicinity. This be- 
ing the first time running the installation over a full 2 hours we realized that we needed to shift the mirrors every half hour to follow the sun, which also meant we had to recalibrate the system. An improved server might control a global motor, which spins the entire installation according to the sun path, while automatically calibrating the system.

\section{DISCUSSION AND LESSONS LEARNED}

In this project, we began to investigate alternatives to traditional screen-based media architecture, based on reflections of natural light. We believe that these can integrate in a subtle way with the physical architecture, enhancing, but not distracting from it. Light reflections, by contrasting against the shaded 'normal' surface, by their very mechanism create a frameless display [23], and can embed projected content in the environment, connect it to objects, or utilize a large surface. Media artists such as Daniel Rozin [24] with his mirror-series have explored controlled surface deformations to depict images. Jason Bruges utilized water surface reflections in his V\&A Mirror Mirror installation [7]. However, few utilize sun reflections projected on a surface. The closest seems [22], where caustic images are deliberately created by surface manipulation. This method of projected image generation has proven usable for larger installations, such as art+com's installation 'River is ...' [2]. Similar to the latter two works, we explored reflections as alternative forms of a pixel, focusing on the threshold between pixel and non-pixel. Due to the goal to create a prototype for a busy urban public space with interaction with passersby, the ornamental non-pixel aspect was only partially translated into the final working prototype. For this location, we focused on natural light reflections with a strong visual impact compared to the delicate light patterns that would blend in better with a calmer space and homogenous material surface.

The basic setting for all installations consisted of an array of digitally controllable reflective surfaces changing contrast. This made the effects ephemeral and required a wellbalanced and dynamic setting of the reflectors, constantly reacting to the sun's changing position. A suitable setting for the northern hemisphere could be an urban square enclosed by a building on the south side and a number of reflectors installed on the north side, projecting onto the north facing and thus shaded facade. This way the reflector is exposed to the sun while directing the sunlight onto the shaded façade. Installations were extremely reliant on the sun and weather when based on natural light. An installation might thus either only be visible at a certain time of day (but this could be desired, like the chimes of a bell tower), or could automatically follow the sun (e.g. using a heliostat mechanism). Furthermore, weather is an intervening factor as such an installation does not work with a cloudy sky. While this could be seen as a lack of control, such weatherbased variations could also contribute an aesthetic element.

While surface-deforming explorations scored higher on aesthetics and ambience, controllable flat mirrors provided more precision regarding the resulting imagery and its control. Specular flat mirrors create high contrast pixels in shaded areas. Overlapped pixels enhanced this effect. Depending on the quality of the mirrors and the projection distance the reflections' edges were sharp or became blurred. Aesthetic effects could be created via movement patterns of controllable reflection dots (cf. [1]). These need to move very smoothly while being noticeable (in particular if there is no on/off shutter mechanism which would allow displacing the pixel unnoticably). We found that the movement has to be rather slow for the human eye to be able to follow it, and the further the projection is away, the slower the motor needs to move. On the other hand, an informational light projection based on mirrors requires a high level of precision and extensive re-calibration whenever the light source or the mirrors move. Reflections with an aesthetic effect (created by surface deformation) are more robust, as they do not necessarily function as a precise pixel conveying concrete information. Such ambient reflections might be suited for deeply shaded areas to bring in light and to animate a surface or create atmosphere. They resemble natural light phenomena such as reflections from water surfaces or ice, and could take ornamental function. A disadvantage of the surface deformation approach is that the scattering of light diffuses it, and reflections get dimmer over a distance. This approach therefore works best at shorter distances, whereas the mirror-approach, focusing the light, works well over longer distances (more than 20m) at daylight.

On a technical level, the precision of manufacturing is a big challenge. Balancing physical forces is not easy as most mechanisms combine different materials and dynamic systems require high engineering effort. A designerly approach does not get far when, as with the puddle vortex, detailed measurements need to be conducted and exact properties specified in order to create a clean vortex. On a process level, we experienced that it is important to test prototypes with actual sunlight. Sometimes we used a mobile phone flash or an office lamp, which create a very different effect. Luminaires that create very focused yet homogenous light beams were most suitable as substitute for sunlight (e.g.slide projectors or a profile spotlight).

We hope readers have been inspired to explore the threshold between pixel and non-pixel with different mechanisms and materials. There is a great potential for sun projections that blend between aesthetic ornament and information source, achieving visual impact in public spaces. An open question to researchers in Media Architecture is how the use of natural (instead of artificial) light influences observers' appreciation, involvement, acceptance, attention, and processing of information. Developing improved technical solutions and prototypes can enhance control of effects, thus extending the range of applications and means of expression based on natural light. Exploration of combinations of different methods of projecting content from natural light could lead to further findings and appealing effects. 


\section{REFERENCES}

1. ART + COM. MOBILITY - REFLECTIVE KINEMATRONIC II, 2010 (see http://artcom.de/?post type $=$ project $\& p=1048$ and $\mathrm{http}: / /$ vimeo.com/67559505 both seen 27.1.2015)

2. ART+COM. River is ... (2012). https://artcom.de/en/project/river-is/ (seen 24.4.2015)

3. Assocreation $2014 \mathrm{http}: / / \mathrm{www}$.assocreation.com/solarpink-pong-in-dubai-uae/ (artist collective) (seen 27.1.2015)

4. Bauer, T. Mediatisation of Architecture. In: Pop, S. et al, 2012, 60-65

5. Blinn, J.F. What is a pixel? Computer Graphics and Applications, IEEE, vol.25, no.5, 2005, 82-87,

6. Brandi, U. and Geissmar-Brandi, C. Lightbook: The Practice of Lighting Design: Planning, Techniques, Realization, Examples. Birkhäuser, Basel, 2001

7. Bruges J. Mirror, Mirror. 2009. http://www.jasonbruges.com/projects/ukprojects/mirror-mirror (seen 24.4.2015)

8. Daalsgard, P. and Halskov, K. Designing Urban Media Façades: Cases and Challenges. In Proc. CHI '10, ACM (2010) 2277-2286.

9. Fox, M; Kemp, M. Interactive Architecture. Princeton Architectural Press, NY. 2009

10. Haeusler, M.H. Media-Augmented Surfaces. Proc. Of eCAADe 2009, p. 483-490

11. Haeusler, M. H. Media Facades - Quo Vadis in Pop, S. et al, 2012, 180-185

12. Hecht, E. Optics -Fourth Edition. Addison Wesley, San Francisco, 2002.

13. Kim, H., Lee, W. Shade Pixel. Proc. of SIGGRAPH '08 posters, ACM (2008) article No.34

14. Klanten, R., Hübner, M. Urban interventions: personal projects in public spaces. Berlin: Gestalten (2010).
15. Lee, J. H. Wall-Facade as Surface. C3, Theme issue 'Wall-Facade as Surface -Towards the Construct of Mediative Facades. C3 Publishers, issue 324, 2010, 132-133

16. Livingston, J. Designing with light: the art, science, and practice of architectural lighting design; Wiley 2014

17. Lowther, C., Schultz, S. Bright: architectural illumination and light installations. Amsterdam: Frame Pub 2008.

18. Lyon, R.F. A brief history of ,pixel' Digital Photography II, IS\&T/SPIE Symposium on Electronic Imaging 2006

19. Moere, A. V., Wouters, N. The role of context in media architecture. Proc. of PerDis'12, ACM: NY 2012, Article 12

20. Moloney, J. Designing kinetics for architectural facades: state change. Routledge, New York, 2011.

21. Neumann, D., Champa, K. Architecture of the night: the illuminated building. Munich New York: Prestel 2002.

22. Papas, M., et al. Goal-based Caustics. Eurographics, 30(2), (2011).

23. Pinhanez, C., Podlaseck, M. To Frame or Not to Frame: The Role and Design of Frameless Displays in Ubiquitous Applications. Proc. Of UbiComp'05. Springer 2005.

24. Rozin, D. Wooden Mirror. 1999. http://www.smoothware.com/danny/ (seen 24.4.2015)

25. Pop, S., Tscherteu, G., Stalder, U., Struppek, M. Urban Media Cultures, avedition, Ludwigsburg, 2012

26. Schumacher, M., Schaeffer, O., Vogt, M.-M. Move Architektur in Bewegung: Dynamische Komponenten und Bauteile. Birkhäuser, Basel, 2010

27. Stubbs, M. The Conceptualisation of Urban Media FACT's Disco Window. In: Pop, S. et al 2012, 78-83 